Ciencia y Educación, Vol. 4, No. 2, mayo-agosto, 2020

ISSN (impreso): 2613-8794・ISSN (en línea): 2613-8808

DOI: https://doi.org/10.22206/cyed.2020.v4i2.pp103-114

\title{
Profesores universitarios y la responsabilidad social de la ciencia
}

\author{
University faculty and the social responsibility \\ of science
}

Ana Hirsch Adler ${ }^{\text {a }}$ ORCID: 0000-0003-4290-4270

Recibido: $17 / 01 / 2020 \bullet$ Aprobado: 23/02/2020

Cómo citar: Hirsch Adler, A. (2020). Profesores universitarios y responsabilidad social de la ciencia. Ciencia y Educación, 4(2), 103-114. Doi: https://doi.org/10.22206/cyed.2020.v4i2.pp103-114

\section{Resumen}

El propósito del artículo es dar cuenta de las principales funciones y actividades que llevan a cabo los profesores e investigadores de posgrado de la Universidad Nacional Autónoma de México (UNAM) con respecto a la responsabilidad social de la ciencia. Se trata de un campo temático significativo tanto para las instituciones de educación superior, como para la sociedad de la que forman parte, por las diversas articulaciones e impactos que se producen entre estas dos instancias. Lo que se presenta forma parte del Estudio sobre la Excelencia del Profesorado del Posgrado de la UNAM y se construye con base en dos fuentes de información: la revisión de documentos internacionales y la realización de entrevistas a 21 académicos universitarios. El trabajo destaca en que a pesar de las variadas tareas que llevan a cabo, que implican una fuerte dedicación, logran, desde sus propios campos de conocimiento, aportar elementos significativos a la sociedad, a través de conocimientos, programas, estrategias y soluciones a problemas relevantes.

Palabras clave: responsabilidad del profesor; ciencia; investigación científica; educación superior; México.

\begin{abstract}
The purpose of the article is to account for the main functions and activities carried out by postgraduate professors and researchers at the National Autonomous University of Mexico (UNAM) with respect to the social responsibility of science. It is a significant thematic field both for higher education institutions and for the society of which they are a part, due to the different articulations and impacts that occur between these two instances. What is presented is part of the Study on the Excellence of the Graduate Teaching Staff of the UNAM and is built based on two sources of information: the review of international documents and the conduct of interviews with 21 university academics. The work stands out in that despite the various tasks that they carry out, which imply a strong dedication, they manage, from their own fields of knowledge, to contribute significant elements to society, through knowledge, programs, strategies and solutions to problems relevant.
\end{abstract}

Keywords: teacher responsibility; science; scientific investigation; higher education; Mexico.

a Universidad Nacional Autónoma de México, México
Correos-e: hirsch@unam.mx, anaha007@yahoo.com.mx 


\section{Introducción}

Aunque en el tema de la responsabilidad social de la ciencia los documentos priorizan las ciencias naturales y exactas, también tratan acerca de las ciencias sociales y del aporte de las humanidades, particularmente en términos de la cultura.

El artículo se divide en dos partes. La primera es una revisión de literatura especializada sobre la responsabilidad social de la ciencia. La segunda da cuenta de lo que manifestaron 21 profesores e investigadores de posgrado de la Universidad Nacional Autónoma de México (UNAM), en relación a ocho aspectos: contribución al conocimiento y al desarrollo de la ciencia; la relevancia de la formación universitaria; formación de niños y jóvenes; los investigadores como profesores y tutores; investigación para la solución de problemas; valores de la ciencia; participación en empresas y diseño de metodologías, prototipos, patentes y modelos, y divulgación y apoyo a los tomadores de decisiones.

\section{Marco de referencia}

En Hirsch (2015) se exponen las principales funciones sociales de la investigación que señalaron los cuarenta coordinadores de posgrado de la Universidad Nacional Autónoma de México, entrevistados en 2009, y catorce profesores de tres universidades de Valencia, España, en 2011. Las principales categorías que se construyeron son: encontrar soluciones útiles para la sociedad, formar recursos de alto nivel, crear nuevos conocimientos, poner el saber al alcance de los demás, detectar problemas que requieren de soluciones, elaborar propuestas, contribuir a la cultura científica del país y preservar la cultura.

La información obtenida con base en la revisión de literatura especializada sobre la responsabilidad social de la ciencia se clasificó en nueve asuntos relevantes: la investigación se lleva a cabo a nombre de la sociedad y con ella; la responsabilidad social es una parte esencial de la conducta responsable de la investigación; los científicos, además de la investigación, llevan a cabo distintas actividades; la generación del conocimiento es la principal responsabilidad; la importante labor de la educación en la formación de investigadores; los investigadores como profesores y tutores; los investigadores como asesores en asuntos públicos; su contribución como parte de comités académicos y tomar en cuenta los valores de la ciencia.

\section{La investigación se lleva a cabo a nombre de la sociedad} y con ella

En cuanto a la cuestión de la ciencia socialmente responsable, Bird (2014) examina las expectativas que tienen los miembros de la sociedad que no pertenecen a una determinada profesión. Por ejemplo, el Uppsala Code of Ethics for Scientists (en Bird, 2014) manifiesta la preocupación por temas de salud, seguridad y bienestar del público y el medio ambiente, y el desacuerdo con la investigación armamentista y de otros estudios que tienen consecuencias negativas en el presente y para el futuro.

Resnik, Elliot y Miller (2015) abordan una cuestión novedosa, ya que denominan "ciencia ciudadana" a la colaboración entre los no científicos y los investigadores profesionales. Exponen que los ciudadanos contribuyen en muchos aspectos, tales como: recopilación de información; diseño de estudios; reclutamiento de sujetos; apoyo para conseguir financiamientos; revisión y planteamiento de normas, reglamentos y leyes; interpretación de información en la investigación comunitaria; publicaciones y proyectos educativos. Esta idea permite reconocer la enorme contribución que la sociedad proporciona a la investigación científica.

La responsabilidad social es una parte esencial de la conducta responsable de la investigación

Para Shamoo y Resnik (2015), la base de la responsabilidad social en la ciencia es doble. Por un lado se debe evitar el daño, y por el otro, se debe beneficiar a los demás. Aquí se plasman dos de los principios generales de la ética de la investigación que son el de "Beneficiencia" y el de "No Maleficencia" (Beauchamp y Childress, 2019).

Resnik y Elliot (2015) afirman que reconocer los propios deberes sociales como científicos es un paso importante para ejercer la responsabilidad social. Plantean tres argumentos sobre su obligación: evitar el daño, ayudar a los otros y reconocer el apoyo y financiamiento por parte de los gobiernos en la educación y en la investigación. 
Indican varias maneras en que los científicos pueden interactuar con el público. Por ejemplo, discutir la implicaciones de la investigación en artículos académicos, información a través de la prensa, cursos universitarios, participar como expertos en la toma de decisiones, apoyar a organizaciones no gubernamentales que centran su trabajo en la ciencia y en la tecnología, promover públicamente políticas específicas e informar de conductas no éticas en la industria, el gobierno y la academia.

El artículo de Resnik y Elliot (2015) finaliza con cinco recomendaciones para evitar problemas cuando se trabaja cercanamente con grupos de la sociedad: colaborar con investigadores que tienen experiencia en políticas públicas, divulgar y discutir los supuestos valorativos que se presentan en temas controvertidos, discutir temas éticos sobre la responsabilidad social, retomar los códigos de conducta y contribuir en la creación de paneles de consejeros y colaboradores por parte de organizaciones científicas, agencias gubernamentales e instituciones que se relacionan con políticas de la ciencia.

Los cientificos, además de la investigación, llevan a cabo distintas actividades

Aunque solo con fines analíticos separamos las funciones que llevan a cabo los investigadores, los autores consultados indican que son diversas las tareas. Damos un ejemplo:

Los científicos tienen obligaciones éticas de beneficiar a la sociedad conduciendo investigaciones que avancen el conocimiento o produzcan resultados útiles (o ambos); educando al público sobre su investigación a través de conferencias; con entrevistas en los medios de comunicación, sitios de internet y libros populares; desarrollando guías nutricionales y de salud, proveyendo de testimonio en procedimientos legales o en comités gubernamentales, o contribuyendo con opiniones informadas en los debates de política pública (Shamoo y Resnik, 2015, p. 283).

La generación del conocimiento es la principal responsabilidad

En el Programa en Pro de la Ciencia: Marco General de Acción (UNESCO, 1999) se hace refe- rencia directa al compromiso de hacer progresar los conocimientos y a la función fundamental de la investigación.

Bird (2014) considera que la tarea más importante es la generación de nuevo conocimiento. Prioriza el desarrollo de investigación precisa y confiable que sea de utilidad para otros investigadores. Especifica el manejo de los datos, los problemas éticos, el trato a los participantes, las preocupaciones de autoría, las prácticas de publicación y los conflictos de interés.

Krogsgaard-Larsen, Thorstrup y Besenhachr (2011, p. 10738), coinciden al indicar que la principal meta de la ciencia es crear nuevo conocimiento. Implica que los encargados de dicha tarea deben desarrollar un nuevo modo de pensar y recobrar la confianza de la sociedad y especifican que "es responsabilidad de los científicos, de todos los sectores de la ciencia, posicionar y definir sus actividades de investigación en el contexto en que puedan contribuir al mejoramiento de la sociedad y a enfrentar los grandes retos de nuestro tiempo".

La Universitat Pompeu Fabra (2018), que forma parte de una Confederación de Sociedades Científicas de España, explicita que la principal labor de la ciencia es la construcción de conocimiento verificable. Manifiesta que "cuando los científicos transmiten el conocimiento a la sociedad, contribuyen a generar ideas y conceptos y esto ayuda a las personas a vivir (...) en una sociedad global con mayor racionalidad, seguridad y libertad" (p. 135).

\section{La importante labor de la educación en la formación} de investigadores

En el Programa en Pro de la Ciencia: Marco General de Acción (UNESCO, 1999) se indica que las universidades deben centrarse en la educación y en la investigación y en su necesaria articulación:

Las universidades han de velar porque sus programas en todos los campos científicos se centren tanto en la educación como en la investigación, y en la sinergia entre ambas, y porque la investigación sea parte integrante de la educación científica. La educación de los científicos debe comprender la adquisición de aptitudes de comunicación y de conocimientos 
básicos relativos a las ciencias humanas.

(UNESCO, 1999, p. 3. Declaración 10)

Borsen (2006), recupera del mismo Programa en Pro de la Ciencia (UNESCO, 1999) la recomendación de que los principios éticos básicos y las responsabilidades de la ciencia sean una parte integral de la educación y de la preparación de todos los científicos. El modelo presenta tres niveles de interacción: el normativo, que establece, discute y justifica los principios éticos de la ciencia; el individual, en donde los principios éticos se traducen en acciones responsables, y el estructural o contextual, que se centra en las instituciones sociales.

Bird (2014, p. 2), en la tarea educativa, se refiere por un lado a los estudiantes y por el otro a los ciudadanos, esto último "con el fin de promover y facilitar las decisiones informadas y la democracia”.

Sobre este mismo asunto, Schuurbiers (2018) complementa lo anterior al decir que aumentan significativamente las oportunidades de incrementar la responsabilidad social en la investigación al ampliar el conocimiento, las habilidades y las actitudes de los investigadores. Agrega que los cambios en la educación son necesarios para contrarrestar las conductas no éticas, por lo que se ha expandido la inclusión de cursos sobre ética en la educación científica, particularmente en las ingenierías y en las ciencias aplicadas.

Por su parte, Mark Frankel (en Pain, 2013), sostiene que los científicos deben involucrase más profundamente en sus responsabilidades sociales; por un lado, al adherirse a los estándares aceptados por la comunidad científica, por otro, al servir a una comunidad más amplia. Plantea tres ideas: a) la ciencia es una institución social, lo que significa que los estudiantes de posgrado deben tener la oportunidad de explorar los valores y expectativas inherentes a sus propias disciplinas y considerar si son consistentes o no con los valores sociales más amplios; b) los jóvenes científicos deben usar sus habilidades para resolver problemas globales, y c) deben tener conciencia de que su educación y sus investigaciones están subsidiadas por la sociedad y tomar en cuenta las expectativas de cómo usar ese conocimiento en el futuro.
La Universitat Pompeu Fabra (2018) incorpora la idea de que se requiere un esfuerzo significativo en la educación y en la difusión de la ciencia para que la sociedad tenga interés y aprecie la naturaleza y los objetivos de la ciencia.

\section{Los investigadores como profesores y tutores}

Varios de los documentos y artículos, por ejemplo Borsen (2006), Institute of Medicine (2009) y Universitat Pompeu Fabra (2018), resaltan el papel que desempeñan los investigadores en su labor como profesores.

Cicerone, Vest y Fineberg en el Prefacio del escrito del Institute of Medicine (2009), señalan que los estudiantes aprenden los estándares de la ciencia al participar en las investigaciones, observan cómo se toman las decisiones y cómo se lleva a cabo la interacción de sus tutores con sus colegas. De esa manera, pueden discutir las prácticas profesionales con sus pares, con el equipo de soporte y con los académicos que cuentan con mayor experiencia. Los asesores y los profesores tienen un efecto profundo en los alumnos. Comentan que los investigadores de mayor experiencia son los que tienen una especial responsabilidad en mantener y promulgar altos estándares en la ciencia y sirven como modelo a seguir para sus alumnos. Añaden que el conocimiento científico se logra colectivamente a través de la discusión y el debate.

\section{Los investigadores como asesores en asuntos públicos}

Otra de las tareas significativas que llevan a cabo los científicos consiste en su participación en diversas discusiones y decisiones y en su intervención en la promoción de políticas públicas sobre el uso apropiado de la ciencia en materias que tienen que ver con temas sociales.

Se da por hecho que "sus capacidades, educación, habilidades, entrenamiento y pericia los equipa para brindar conocimiento especializado y perspectivas para un entendimiento y análisis de asuntos y problemas que afectan a la sociedad de la que son parte" (Bird, 2014 , p. 2). La autora incorpora la idea de que como participantes de estas discusiones los investigadores "pueden ayudar a la construcción de puentes multi- 
disciplinarios e interdisciplinarios que traten acerca de la rendición de cuentas de la investigación públicamente financiada” (Bird, 2014. p. 2).

Shamoo y Resnik (2015) opinan que se les solicita a los científicos su testimonio experto en muchas materias, tales como casos judiciales y comités gubernamentales y que proveen su conocimiento y experiencia en lo que concierne a su área de conocimiento y a sus proyectos de investigación.

Elliot y Resnik (2014) afirman que los investigadores deben estar en abierta comunicación con los encargados de proponer políticas que conciernen a la ciencia y buscar que esta sea transparente sobre la manera en que los intereses y valores influyen en los razonamientos.

\section{Su contribución como parte de comités académicos}

Otro aspecto relevante, es el involucramiento de los académicos en comités institucionales y departamentales que tienen que ver con el reclutamiento, contratación, promoción y otorgamiento de plazas, ya que el profesorado "tiene la responsabilidad de asegurar que todos los miembros sean no solamente buenos investigadores sino también educadores efectivos y buenos mentores para sus estudiantes" (Bird, 2014, p. 3). También inciden, por ejemplo, en la evaluación de las publicaciones, en los comités que financian la investigación y en eventos en que se discuten los resultados.

\section{Tomar en cuenta los valores de la ciencia}

De acuerdo con Bird (2014), lo que orienta el trabajo de los investigadores es la búsqueda de la verdad. Pueden dar cuenta de los valores y supuestos que tienen que ver con los procesos, las preguntas que se hacen, el diseño de los estudios, el análisis de los datos y su interpretación y presentación.

Coincide en ello The Norwegian National Research Ethics Committees (2016) que indica que la obligación fundamental de la ciencia es la búsqueda de la verdad. Las normas y valores científicos, éticos y legales regulan la responsabilidad de la investigación.

La investigación tiene también una responsabilidad social, ya sea instrumental como base para la toma de decisiones sociales, crítica como fuente de correcciones y cursos de acción alternativos o deliberativos, y como proveedores de conocimiento basados en la investigación para el discurso público (p. 11).

De la misma manera, Daniël Schuurbiers (2018) plantea que los logros académicos necesariamente involucran el compromiso valorativo y que sus resultados son significativos desde el punto de vista social. Los valores culturales juegan un papel en la investigación y es tarea de los científicos reflexionar en las metas que dirigen la indagación y el significado potencial de sus resultados y consecuencias. Implica que son dos los requisitos para ampliar la responsabilidad social: la voluntad de los investigadores en reflejar críticamente el contexto socio-ético de su investigación y en la aplicación de los principios normativos a las decisiones concretas del contexto.

La Universitat Pompeu Fabra (2018, p. 135) también se refiere a los valores y a la ética con respecto a que las actividades científicas y tecnológicas: "afectan las ideas colectivas, valores, intereses, preferencias, necesidades y oportunidades en grados diversos".

\section{Metodología}

Este trabajo es parte de la investigación "Estudio sobre la Excelencia del Profesorado del Posgrado de la UNAM" (2015-2020). Su propósito es dar a conocer las principales funciones y actividades que llevan a cabo los académicos de posgrado en referencia al campo temático de la responsabilidad social de la ciencia. Se ubica en lo desarrollado en el programa de entrevistas, específicamente en la pregunta abierta: Uno de los planteamientos que ha cobrado fuerza sobre los fines de la investigación es el compromiso que se debe tener para promover el beneficio social. ¿De qué manera lo considera usted en sus investigaciones? que se aplicó a 21 académicos.

La UNAM clasifica sus 41 posgrados en cuatro áreas de conocimiento: Ciencias Físico-Matemáticas y de las Ingenierías; Ciencias Biológicas, Químicas y de la Salud; Ciencias Sociales y Humanidades y de las Artes. Los entrevistados se eligieron de modo de representar algunos posgrados de las cuatro áreas y de que hubiese un equilibro entre académicos de género masculino y femenino (ver tabla 1). 
Tabla 1. Sujetos de la muestra cualitativa por áreas de conocimiento

\begin{tabular}{|c|c|c|c|c|}
\hline ÁREAS DE CONOCIMIENTO & POSGRADOS & $\begin{array}{l}\text { ENTIDAD DE } \\
\text { ADSCRIPCIÓN }\end{array}$ & $\begin{array}{l}\text { CATEGORÍA } \\
\text { Y NIVEL }\end{array}$ & GÉNERO \\
\hline $\begin{array}{l}\text { C. Físico-Matemáticas y de las } \\
\text { Ingenierías }\end{array}$ & $\begin{array}{l}\text { Ingeniería y Ciencia e } \\
\text { Ingeniería de Materiales }\end{array}$ & Facultad de Ingeniería & Titular B & Masculino \\
\hline $\begin{array}{l}\text { C. Físico-Matemáticas y de las } \\
\text { Ingenierías }\end{array}$ & Ingeniería & Instituto de Ingeniería & Titular C & Masculino \\
\hline $\begin{array}{l}\text { C. Físico-Matemáticas y de las } \\
\text { Ingenierías }\end{array}$ & Ciencias de la Tierra & Instituto de Ingeniería & Asociado C & Femenino \\
\hline $\begin{array}{l}\text { C. Físico-Matemáticas y de las } \\
\text { Ingenierías y C. Biológicas, Químicas } \\
\text { y de la Salud }\end{array}$ & $\begin{array}{l}\text { Ciencias Físicas y Ciencias } \\
\text { Químicas }\end{array}$ & $\begin{array}{l}\text { Instituto de Investigaciones } \\
\text { Nucleares }\end{array}$ & Titular C & Masculino \\
\hline $\begin{array}{l}\text { C. Físico-Matemáticas y de las } \\
\text { Ingenierías }\end{array}$ & Ciencias de la Tierra & Instituto de Geología & Titular C & Femenino \\
\hline C. Biológicas, Químicas y de la Salud & Psicología & Facultad de Psicología & Titular C & Femenino \\
\hline C. Biológicas, Químicas y de la Salud & Psicología & Facultad de Psicología & Titular C & Masculino \\
\hline C. Biológicas, Químicas y de la Salud & Ciencias Bioquímicas & Facultad de Química & Titular C & Femenino \\
\hline C. Biológicas, Químicas y de la Salud & Ciencias Biomédicas & $\begin{array}{l}\text { Instituto de Fisiología } \\
\text { Celular }\end{array}$ & Titular C & Masculino \\
\hline C. Biológicas, Químicas y de la Salud & Ciencias Químicas & Facultad de Química & Titular C & Masculino \\
\hline C. Biológicas, Químicas y de la Salud & $\begin{array}{l}\text { Ciencias Biomédicas } \\
\text { y Ciencias Médicas, } \\
\text { Odontológicas y de la Salud }\end{array}$ & $\begin{array}{l}\text { Instituto de Investigaciones } \\
\text { Biomédicas }\end{array}$ & Titular B & Masculino \\
\hline Ciencias Sociales & $\begin{array}{l}\text { Estudios Latinoamericanos } \\
\text { y Trabajo Social }\end{array}$ & $\begin{array}{l}\text { Centro de Investigaciones } \\
\text { Interdisciplinarias en } \\
\text { Ciencias y Humanidades }\end{array}$ & Titular C & Femenino \\
\hline $\begin{array}{l}\text { Ciencias Sociales y C. Biológicas, } \\
\text { Químicas y de la Salud }\end{array}$ & $\begin{array}{l}\text { Ciencias Políticas y Sociales } \\
\text { y Psicología }\end{array}$ & $\begin{array}{l}\text { Centro de Investigaciones } \\
\text { Interdisciplinarias en } \\
\text { Ciencias y Humanidades } \\
\end{array}$ & Titular B & Femenino \\
\hline Ciencias Sociales & Ciencias Políticas y Sociales & $\begin{array}{l}\text { Instituto de Investigaciones } \\
\text { Sociales }\end{array}$ & Titular C & Masculino \\
\hline Ciencias Sociales & Ciencias Políticas y Sociales & $\begin{array}{l}\text { Instituto de Investigaciones } \\
\text { Sociales }\end{array}$ & Titular C & Masculino \\
\hline Ciencias Sociales & Derecho & Facultad de Derecho & Titular C & Femenino \\
\hline Humanidades y de las Artes & Historia & $\begin{array}{l}\text { Facultad de Filosofía y } \\
\text { Letras }\end{array}$ & Titular C & Femenino \\
\hline Humanidades y de las Artes & Arquitectura & Facultad de Arquitectura & Titular C & Masculino \\
\hline Humanidades y de las Artes & Filosofía de las Ciencias & $\begin{array}{l}\text { Instituto de Investigaciones } \\
\text { Filológicas }\end{array}$ & Titular C & Masculino \\
\hline Humanidades y de las Artes & Pedagogía & $\begin{array}{l}\text { Instituto de Investigaciones } \\
\text { sobre la Universidad y la } \\
\text { Educación }\end{array}$ & Titular C & Femenino \\
\hline Humanidades y de las Artes & Lingüística & $\begin{array}{l}\text { Instituto de Investigaciones } \\
\text { Filológicas }\end{array}$ & $\begin{array}{l}\text { Adscripción } \\
\text { temporal }\end{array}$ & Femenino \\
\hline
\end{tabular}

Nota: la categoría y nivel más alto es Titular C.

Fuente: elaboración propia con base en las características de la muestra 
Se entrevistó a cinco académicos del área de Ciencias Físico-Matemáticas y de las Ingenierías, seis de Ciencias Biológicas, Químicas y de la Salud, cinco de Ciencias Sociales y cinco de Humanidades y de las Artes, para un total de 21 entrevistas. Estos académicos fueron localizados en bases de datos institucionales y accedieron a contestar el instrumento.

La estrategia para el análisis fue la siguiente: todas las entrevistas fueron grabadas y posteriormente transcritas. De la versión escrita, se fueron separando y eligiendo los principales argumentos que dieron los académicos con respecto a las diversas funciones $\mathrm{y}$ actividades que llevan a cabo dentro de la universidad y fuera de ella, que se relacionan directa o indirectamente con el beneficio a la sociedad. Con dichas explicaciones, se fueron construyendo categorías y se conservaron los testimonios más importantes que propusieron los sujetos — para cada una de ellascon el fin de no perder lo expresado directamente por ellos. Las ocho categorías que se definieron son: contribución al conocimiento y al desarrollo de la ciencia; la relevancia de la formación universitaria; formación de niños y jóvenes; los investigadores como profesores y tutores; investigación para la solución de problemas; valores de la ciencia; participación en empresas y diseño de metodologías, prototipos, patentes y modelos, y divulgación y apoyo a los tomadores de decisiones.

\section{Resultados}

\section{Contribución al conocimiento y al desarrollo de la ciencia}

Los académicos enfatizaron que la generación del conocimiento es en sí un beneficio para la sociedad y que contribuye al acervo del saber universal, a formar parte de comunidades internacionales de investigación, a la utilidad e influencia que tiene la labor que realizan y al desarrollo de la academia. Dijeron que:

Contribuimos a este acervo del conocimiento universal, somos parte de comunidades internacionales que hacen investigación y [...] en particular en el área de la Física (entrevista 4, Posgrado: Ciencias Físicas).

[...] la investigación por sí misma, genera conocimiento, en algún momento va a ser útil para la sociedad [...]. Es algo que en realidad está implícito en la labor de la investigación y más si se trata de ciencia básica y eso es lo que debemos fomentar, si queremos ser buenos investigadores y buenos docentes, nuestra actividad principal debe estar en generar conocimiento nuevo, en probar cosas, en tratar de entender fenómenos (entrevista 5, Posgrado: Ciencias de la Tierra).

Contribuimos al desarrollo de la academia, de la investigación científica, en México y en el extranjero. La otra es que nos hemos concentrado con un tema de investigación interesante [...], que ha tenido bastante influencia en muchos campos, yo he podido influir sobre muchos estudiantes, [...] sí hemos contribuido y publicado cosas muy relevantes [...]. Nosotros lo que hacemos es preguntas básicas que pueden llevar a un proceso, lo hacemos desde la perspectiva básica del conocimiento (entrevista 9, Posgrado: Ciencias Biomédicas).

[...] el contribuir al desarrollo de la ciencia es un beneficio para la sociedad, aunque sea puramente abstracto [...], el pensamiento científico se ha vuelto fundamental, y hay un pensamiento de ciencia básica y de conocimiento filosófico, que tiene una aplicación muy remota para la sociedad, pero en el momento en que enriquece el conocimiento, va a tener a la larga un gran beneficio (entrevista 14, Posgrado: Ciencias Políticas y Sociales). [...] es el beneficio social real del producto, ahí tiene dos vertientes, que sería el conocimiento, la mayor parte del trabajo que yo he hecho en mi vida es más de documentación de que los fenómenos existen, y en ese sentido, fortalecer una teoría o ponerla a prueba, y luego ya en menor cantidad, el beneficio directo a los individuos (entrevista 11, Posgrados: Ciencias Médicas, Odontológicas y de la Salud y Ciencias Biomédicas).

Como podemos ver, hay ideas coincidentes entre lo planteado en los documentos revisados y en lo dicho por los informantes, acerca de la generación de 
nuevo conocimiento. Coinciden en que su creación es la principal responsabilidad social de la ciencia; puesto que, de diversas maneras, contribuye al mejoramiento de la sociedad.

\section{La relevancia de la formación universitaria}

Los profesores e investigadores expresaron que inciden en que los estudiantes construyan conocimiento, a partir de un cambio de mentalidad, de la clarificación de conceptos, de la reflexión, de la argumentación y de buscar perspectivas diversas. Opinaron que la formación de recursos humanos en la universidad apoya no solo a sus estudiantes, sino también a las comunidades de las que provienen.

[...] hago que los estudiantes logren construir conocimiento y pensamiento crítico. Yo creo que el área en la que estoy, el beneficio a la sociedad es muy importante, es esencial, pero no es palpable, es un puente conceptual [...] estamos promoviendo y estamos contribuyendo al acervo cognitivo de una sociedad, [...] es enseñar a pensar bien por medio de la argumentación (entrevista 19, Posgrados: Filosofía, Filosofía de la Ciencias e Ingeniería y Ciencias de la Computación).

Lo considero en cuanto a que mi trabajo tiene que ser comunicable en el sentido de que tiene que aportar a ciertos grupos los elementos para analizar su situación, para que los estudiantes tengan algunos referentes [...] (entrevista 20, Posgrado: Pedagogía).

[...] hay algo que uno está enseñando a hacer a los estudiantes, que es a sofisticar su pensamiento, yo creo que eso no se ve, es difícil de medir, pero es un efecto sobre la sociedad. Los posgrados en Humanidades y Sociales son espacios muy propicios para eso, este país requiere gente que pueda reflexionar, adoptando perspectivas diversas (entrevista 21, Posgrado: Lingüística).

El primer impacto de todas estas investigaciones, es que tu formas gente y les das habilidades a la gente de tu entorno, y la UNAM es la universidad de la nación, aquí viene gente de todos lados de México y de muchas partes del contexto Iberoamericano [...]. Es un impacto importante cuando ellos regresan a sus comunidades, a sus estados, a sus universidades o a sus países de origen (entrevista 10, Posgrado: Ciencias Químicas).

[...] básicamente, esa es la misión de la universidad, servir a la sociedad. Yo creo que, inclusive, cuando uno hace una intervención, ya sea para una recogida de información o algo más [...], el propio cuestionamiento a la persona, la mueve a reflexionar sobre sí, y eso ya es una aportación. [...] desde impulsar un diálogo diferente, una reflexión diferente en el aula, en congresos, en un artículo, estar en contacto con las personas y la devolución de los resultados a los propios participantes (entrevista 6, Posgrado: Psicología).

Yo creo que hemos influido en que haya un cambio de mentalidad en las personas y porque lo digo en plural, porque somos varias las académicas que hemos trabajado para que haya más mujeres en educación superior y en investigación (entrevista 12, Posgrados: Estudios Latinoamericanos y Trabajo Social).

De la primera parte del artículo, acerca de la labor de la educación en la formación de investigadores y de lo expresado por los académicos, podemos resaltar la estrecha integración que existe entre la educación y la investigación en el ámbito universitario. Entre los diversos asuntos están las posibilidades y desafíos, el impacto que tienen para atender las necesidades de la sociedad, la relevancia de una buena preparación de los científicos y de los estudiantes en la ética de la investigación y el vínculo entre conocimientos, habilidades y actitudes.

\section{Formación de niños y jóvenes}

En este rubro, solo ubicamos un testimonio por parte de los académicos. Trata acerca del compromiso y el aporte que se lleva a cabo desde la universidad en la formación científica en niveles previos de la enseñanza y en espacios extracurriculares. Sorprende el hecho de que solo lo haya planteado un profesor de posgrado, ya que la UNAM tiene un amplio programa de extensión universitaria, en la mayoría de las disci- 
plinas, tanto para la propia universidad, como para otros sectores de la sociedad.

Hace dos años iniciamos un programa que se llama PAUTA, que tiene que ver con adoptar un talento, con el cual hemos iniciado trabajando con unos 1,500 o 1,600 niños por año, en cuatro estados de la república, en donde hemos estado haciendo acompañamientos sostenidos. Es un programa educativo complementario de la actividad escolar; hay clubes de ciencia en las escuelas, talleres de ciencia en espacios diferentes de las escuelas y aquí en la UNAM, y así en otros lugares [...]. Es una forma más directa de compartir con la comunidad, retribuir un poco de lo mucho de lo que la universidad nos brinda (entrevista 4, Posgrado: Ciencias Físicas).

\section{Los investigadores como profesores y tutores}

Aunque los académicos priorizan la investigación, dan cuenta también de la importancia que tienen la docencia y las tutorías en la formación de los estudiantes, incluyendo el nivel de la licenciatura. Dijeron lo siguiente:

Hay un beneficio muy directo que hacemos que es enseñar, formar a otros jóvenes (entrevista 4, Posgrado: Ciencias Físicas).

[...] formación de recursos humanos. Yo estoy convencido que mi contribución a ese nivel no es buena, sino muy buena [...] porque conozco dónde han ido, dónde se han desarrollado, dónde están ahora los ex estudiantes del laboratorio, el $90 \%$ de los estudiantes que se han graduado en mi grupo de investigación son investigadores. Entonces, ahí está mi contribución, son personas, son familias que mejoraron su estatus y su condición de vida y académica y decidieron hacer ciencia [...] (entrevista 9, Posgrado: Ciencias Biomédicas).

[...] le dedico tiempo a la licenciatura y tengo muchas tesis. A la docencia le dedico bastante tiempo (entrevista 17, Posgrado: Historia).

En uno de los escritos (Institute of Medicine, 2009) se especifican algunos elementos centrales acerca de la manera en cómo aprenden los alumnos los estándares de la ciencia, primordialmente con base en el trabajo colectivo. También se refieren al significativo impacto que tienen los profesores y los asesores en el aprendizaje, especialmente los que cuentan con una amplia experiencia. De la misma manera, uno de los entrevistados resaltó la contribución en la formación de recursos humanos, señalando su trabajo en el laboratorio y en un grupo de investigación.

\section{Investigación para la solución de problemas}

Los académicos valoraron el compromiso social, el conocimiento de los problemas sociales y la atención a los problemas nacionales en general. También lo hicieron con respecto a temas específicos, tales como: medio ambiente, contaminación, salud, familia y pareja, violencia, derechos humanos y pueblos y comunidades indígenas.

[...] uno trabaja para tratar de mejorar la situación, para aportar algún tipo de solución en relación a los grandes problemas nacionales (entrevista 15, Posgrado: Ciencias Políticas y Sociales).

Hay que enfocar cómo se da el compromiso social [...], todo está enfocado al mejoramiento de la sociedad y al cambio social [...]; trabajamos eso permanentemente. Aquí en el laboratorio, los estudiantes trabajan con comunidades y en los grupos de investigación de posgrado, tratamos de que los temas se enfoquen a entender a la sociedad y los problemas sociales (entrevista 18, Posgrado: Arquitectura).

El segundo impacto que yo veo, es que trato de darle a la investigación un enfoque muy puntual hacia problemas de contaminación, de residuos y de energía, que son problemas hoy en día muy importantes en el mundo y a nivel local (entrevista 10, Posgrado: Ciencias Químicas).

Cada vez que nos planteamos hacer un nuevo tema o desarrollar un proyecto de investigación, platicamos los que formamos parte del Centro que lo que vayamos a investigar sea más amigable con el medio ambiente (entrevista 1, Posgrados: Ingeniería y Ciencia e Ingeniería de Materiales).

Hemos podido desarrollar información que nos dice cómo trabajar con $[\ldots]$ personas para que 
no incurran en conductas de riesgo. [...] en esa misma tendencia de la salud, en los últimos ańos hemos estado trabajando temas que tienen que ver con obesidad y diabetes [...]. Otras áreas que hemos trabajado es la familia y la pareja y sobre la violencia (entrevista 7, Posgrado: Psicología).

[...] últimamente mis investigaciones están enfocadas principalmente al tema de derechos humanos [...] tiene que ver con la honestidad, cuando existe una interpretación de los derechos fundamentales deficiente [...], considero que ese tipo de cuestiones me han permitido devolver un poco a la sociedad. Estoy trabajando, también, con lo que son pueblos y comunidades indígenas, (entrevista 16, Posgrado: Derecho).

[...] yo creo que para mí el beneficio más importante que tiene la ciencia aplicada es la crítica social [...] (entrevista 14, Posgrado: Ciencias Políticas y Sociales).

Como puede notarse, son muchos los problemas sociales que dieron a conocer los sujetos. Resalta el hecho de que las soluciones que consideran que son útiles para la sociedad, provienen directamente de las investigaciones que llevan a cabo en sus áreas de conocimiento.

\section{Valores de la ciencia}

Se especificaron dos valores de la ciencia: búsqueda de la verdad y hacer el trabajo de la mejor manera.

[...] a la lectura le dedico mucho tiempo [...] tiene que ver con la verdad, [...] es muy importante desentrańar, abrir la verdad y que la sociedad lo conozca. Yo pienso junto con unos colegas, que hay que poner el dedo en el reglón, y decir cuál fue la verdad [...]. Tenemos que emprender investigaciones nuevas (entrevista 17, Posgrado: Historia).

[...] yo hago realmente ciencia básica, no hago cosas aplicadas que se puedan usar a futuro, pero yo creo que aquí hay una cuestión ética de honestidad muy grande, porque yo lo que sé hacer es eso, ciencia básica, yo lo que puedo ofrecer son los datos que estoy viendo [...] me limito a hacer mi trabajo de la mejor manera (entrevista 8, Posgrado: Ciencias Bioquímicas).
[...] el equipo que hace la investigación y la formación de los individuos [debe] aprender a hacer el trabajo bien hecho, el ser consciente de la responsabilidad ética que uno tiene de reportar lo que encuentra [...] (entrevista 11, Posgrados: Ciencias Médicas, Odontológicas y de la Salud y Ciencias Biomédicas).

Del marco de referencia, retomamos el rubro sobre tomar en cuenta los valores de la ciencia, ya que, Bird (2014) y The Norwegian National Research Ethics Committees (2016) resaltan la búsqueda de la verdad. Los participantes, además, insistieron en la necesidad de llevar a cabo un buen trabajo y se hizo mención al valor de la honestidad.

Participación en empresas y diseño de metodologías, prototipos, patentes y modelos

Resulta de gran interés, que además del intenso trabajo que los académicos llevan a cabo al interior de la universidad, contribuyan con diversos tipos de organizaciones y que generen productos derivados de la investigación. Por ejemplo:

Hay otras empresas en las hemos participado, en las que hemos intentado contribuir. Gran parte del trabajo de la línea que coordino, es muchos trabajos hacia afuera [...]. También hacemos investigación, nos metemos en el desarrollo de los modelos [...] (entrevista 3, Posgrado: Ingeniería ambiental).

Hay muchas oportunidades si usa uno la imaginación. Hemos construido prototipos y se han conseguido patentes para diferentes aplicaciones (entrevista 2, Posgrado: Ingeniería).

[...] hago metodologías [...] tenemos por ahí, incluso, varias patentes (entrevista 10, Posgrado: Ciencias Químicas).

Hemos desarrollado modelos bastante complejos bio-psico-culturales, que lo que van a hacer es identificar todas aquellas cosas que las personas pueden hacer para mejorar su vida (entrevista 7 , Posgrado: Psicología).

La mayoría de los participantes indicaron que se dedican fundamentalmente a las ciencias básicas, pero hay desde luego aplicaciones importantes que llevan a cabo los investigadores. 
Divulgación y apoyo a los tomadores de decisiones

Únicamente en dos de los posgrados se hizo referencia a la función de divulgación de la información y a la contribución en asuntos públicos.

Una de las cosas que debemos hacer es la divulgación y promoción de la información lo más posible (entrevista 7, Posgrado: Psicología).

En ese sentido, lo que uno hace ayuda a que los tomadores de decisiones puedan hacerlo de manera informada (entrevista 14, Posgrado: Ciencias Políticas y Sociales).

Los autores y documentos que se tomaron en cuenta en la primera parte del artículo resaltan como una de las tareas significativas la participación de los investigadores en foros públicos y en la promoción de políticas públicas. Los académicos mencionaron limitadamente este tema.

\section{Conclusiones}

Se cumplió el propósito específico de este trabajo, que consistió en dar a conocer las principales funciones y actividades que llevan a cabo los académicos de posgrado en referencia al campo temático de la responsabilidad social de la ciencia; considerando fundamentalmente lo que ellos expresaron cuando respondieron a la pregunta abierta sobre este asunto. Las principales funciones fueron: contribución al conocimiento y al desarrollo de la ciencia; formación de los estudiantes; formación de niños y jóvenes; su rol como profesores y tutores; la solución de problemas; la consideración de los valores de la ciencia; la participación en empresas y diseño de metodologías, prototipos, patentes y modelos, y divulgación y apoyo a los tomadores de decisiones.

Es importante agregar, en relación con las múltiples tareas que realizan, que su cometido es cada vez más dinámico y demandante, ya que se han ido incluyendo nuevas obligaciones, tanto en cada una de las dependencias universitarias como en las instituciones en general, y han aumentado fuertemente los requisitos de los órganos financiadores para la aprobación de proyectos de investigación a nivel nacional e internacional.

Una consideración relevante, al respecto, es la reflexión acerca de la compleja articulación de tres elementos: la distribución del tiempo, el esfuerzo que se emplea y los recursos con que cuentan para poder atender la diversidad de ocupaciones en las que laboran.

Autores como Bak y Han (2015); Palali, Van Elk, Bolhaar y Rud (2017) y Vidal y Quintanilla (2000) con base en la investigación empírica, señalan que los científicos generalmente priorizan la investigación, en mayor grado que con respecto a las tareas de docencia y tutorías. Esto se debe, en gran medida, a que las universidades destinan mayores recursos para la investigación, lo que genera que los académicos le dediquen más tiempo y más esfuerzo a esta función sustantiva, y porque los logros generados por la investigación se han vuelto de mayor relevancia para la promoción académica. Mencionan la enorme influencia que tienen los programas de incentivos en los proyectos de investigación. Además, argumentan que la investigación promueve la mejora de la capacidad de los profesores, especialmente en cuanto a mantenerlos actualizados en los avances de sus disciplinas.

Los profesores e investigadores entrevistados dieron cuenta de acciones relevantes que llevan a cabo con respecto al papel que cumplen las ciencias y las humanidades en diversos estratos de la población. Parecería entonces, que si la principal preocupación se focaliza en la investigación, los académicos tenderían a dejar de lado las acciones que buscan beneficiar a la sociedad, y sin embargo, logran articular sus proyectos de investigación con interesantes propuestas que la favorecen.

\section{Referencias}

Bak, H. y Han, K. (2015). Too much Emphasis on Research? An empirical examination of the relationship between research and teaching in multitasking environments. Research in High Education, 56(8), 843-860. Doi:: https://doi. org/10.1007/s11162-015-9372-0.

Beauchamp, T. y Childress, J. (2019). Principles of Biomedical Ethics. [8th ed.]. New York, United States: Oxford University Press.

Bird, S. (2014). Socially Responsible Science is More than "Good Science". JMBE Journal of Microbiology \& Biology Education, 15(2), 169-172. Doi: 10.1128/ jmbe.v15i2.870. 
Borsen, T. (2006). Academic and Social Responsibility of Scientists. Journal of Science and World Affairs, 2(2),71-92. Recuperado de http://www.icsu.org/ Gestion/img/ICSU_DOC_DOWNLOAD/ synthesis02/output/ists_mexico_consensus.pdf.

Elliot, K.C. y Resnik, D.B. (2014). Science, Policy and Transparency of Values. ehp Environmental Health Perspectives 122, 647-650; Doi: 10.1080/ 08989621.2014 .1002608

Hirsch, A. (2015). Funciones sociales y desafíos para las universidades según profesores en México y en España. En O. E. Arango, J.E. Moncayo y J.J Martí-Noguera, (eds). Experiencias Iberoamericanas en Responsabilidad Social Universitaria, pp. 224-248. Medellín, Colombia, Luis Amigó Fundación Universitaria.

Institute of Medicine (2009). On Being a Scientist: A Guide to Responsible Conduct in Research. (3 ${ }^{\text {rd }}$ ed.). Washington, D.C, Estados Unidos: The National Academies Press. Doi: https://doi. org/10.172226/12192.

Krogsgaard-Larsen, P., Thorstrup, P. y Besenhacher, F. (2011). Scientific Social Responsibility: A Call to Arms. GDCH A Journal of the German Chemical Society. Germany: Wiley Online Library. Doi: https://doi.org/10.1002/anie. 201105641

Pain,E.(2013). TheSocialResponsibilitiesofScientists. Science. Recuperado de http://www.sciencemag. org/02/social-responsabilities-scientists

Palali, A., Van Elk, R., Bolhaar, J. y Rud, I. (2017). "Are Good Researchers also Good Teachers? The relationship between research quality and teaching quality". La Haya, discussion paper 347, CPB Netherlands, Bureau for Economic Policy Analysis. Recuperado de: https://www. cpb.nl/sites/default/files/omnidownload/ cpb-discussion-paper-347-aregood-researchers-also-good-teachers.pdf
Resnik,D.B.yElliot,K.C.(2015). TheEthicalChallenges of Socially Responsible Science. Accountability in Research Policies and Quality Assurance, 23(1). Doi: 10.1080/08989621.2014.1002608

Resnik, D.B., Elliot, K.C. y Miller, A.K. (2015). A framework for addressing ethical issues in citizen science. Environmental Science and Policy. Published by Elsevier Ltd. Recuperado de http://www.elsevier.com/locate/envsci

Shamoo, A.E. \& Resnik, D.B. (2015). Chapter 12: Science and Social Responsibility. Responsible Conduct of Research (3 ${ }^{\text {rd }}$. ed.), [pp. 283-310]. New York, United States: Oxford University Press.

Schuurbiers, D. (2018). Social Responsibility in Research Practice. Engaging applied scientists with the socio-ethical context of their work, PHD Thesis. Technische Universiteit Delft, Netherlands. Recuperado de http://proeffabriek.nl/wp-content/ uploads/2018/01/Thesis_D_Schuurbiers_Social_ Responsibility_in_Research_Practice.pdf

UNESCO (1999). Conferencia Mundial sobre la Ciencia: Programa en Pro de la Ciencia: Marco General de la Acción. Budapest. Recuperado de: http://unesco.org/science/wcs/esp/marco_ accion_s.htm

The Norwegian National Research Ethics Committees (2016). Guidelines for Research Ethics in the Social Sciences, Humanities, Law and Theology. Recuperado de http://www.etikkom.no

Universitat Pompeu Fabra (2018). Science for Society: the Social Responsibility of Scientists. Barcelona, España: Confederación de Sociedades Científicas de España. Recuperado de: http://www.upf.edu/ pcstacademy/_docs/cosce_en_02

Vidal, J. y Quintanilla, M (2000). "The Teaching and Research Relationship within and Institutional Evaluation", Higher Education, 40(2), 217-229, Recuperado de http://www.jstor.org/stable/ 3448111 